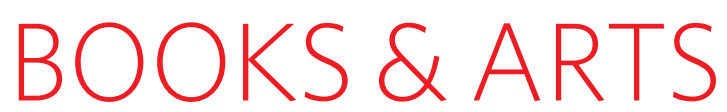

\title{
Cool is not enough
}

\section{There's more to life than the second law of thermodynamics.}

\section{Into the Cool: Energy Flow, \\ Thermodynamics and Life \\ by Eric D. Schneider \& Dorion Sagan \\ University of Chicago Press: 2005. 362 pp. \\ $\$ 30, £ 21$}

\section{J. Doyne Farmer}

The level of organization in even the simplest living systems is so remarkable that many, if not most, non-scientists believe that we need to go outside science to explain it. This belief is subtly reinforced by the fact that many scientists still think the emergence of life was a fortuitous accident that required a good roll of the molecular dice, in a place where the conditions are just so, in a Universe where the laws of physics are just right.

The opposing view is that matter tends to organize itself according to general principles, making the eventual emergence of life inevitable. Such principles would not require any modifications of the laws of physics, but would come from a better understanding of how complex behaviour arises from the interaction of simple components.

Complex organization is not unique to living systems: it can be generated by very simple mathematical models, and is observed in many non-living physical systems, ranging from fluid flows to chemistry. Self-organization in non-living systems must have played a key role in setting the stage for the emergence of life. Many scientists have argued that certain principles of complex systems could explain the emergence of life and the universal properties of form and function in biology, and perhaps even provide insights for social science. The problem is that these principles have so far remained undiscovered.

In their book Into the Cool, Eric Schneider and Dorion Sagan claim that non-equilibrium thermodynamics provides the key principle that has been lacking. They review its application to topics ranging from fluid dynamics and meteorology to the origin of life, ecology, plant physiology, and evolutionary biology, and even speculate about its relevance to health, economics and metaphysics. The book contains a wealth of good references and is worth buying for this reason alone.

When the discussion sticks to applications where thermodynamics is the leading actor, such as the energy and entropy flows of the Earth, or the thermodynamics of ecological

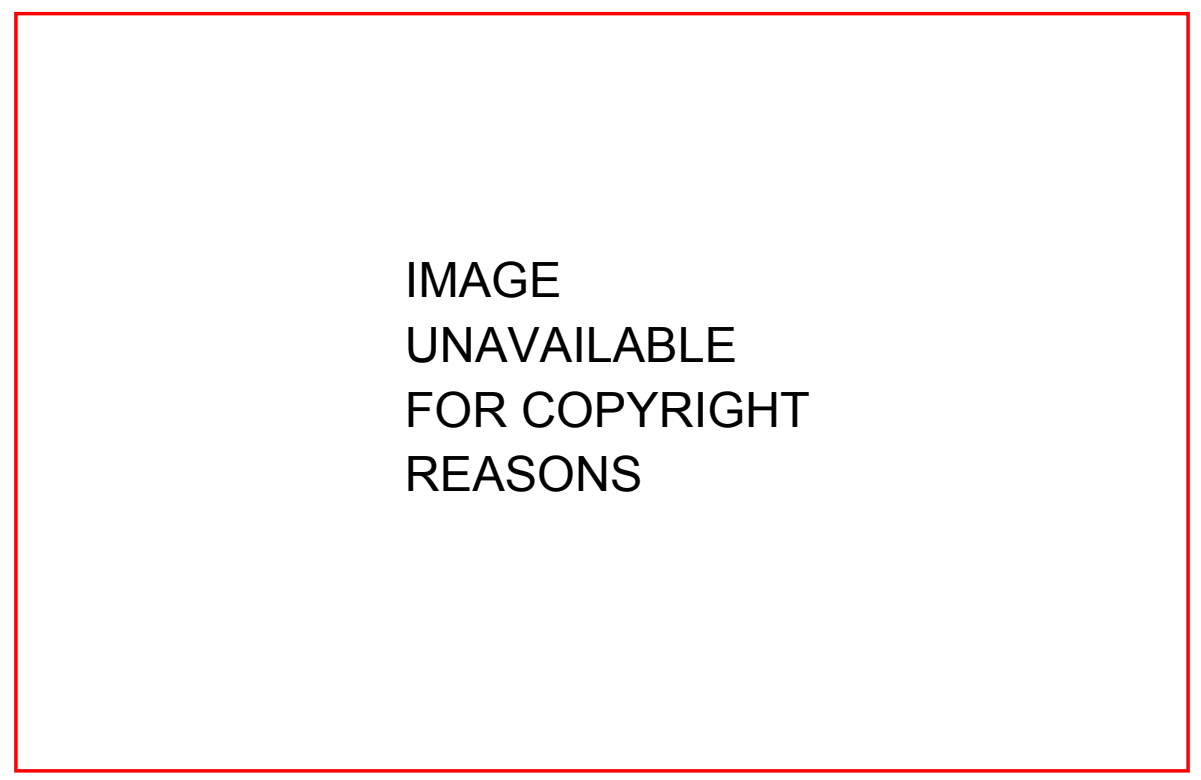

A complex problem: can a need to reduce energy gradients help to drive the evolution of forests?

systems, it is informative and worthwhile, but it is repetitive and seems disorganized in places.

The book is less successful as an exposition of a grand theory. It gets off to a bad start on the dust-jacket, which says: "If Charles Darwin shook the world by showing the common ancestry of all life, so Into the Cool has a similar power to disturb - and delight." While it may be wise to stand on the shoulders of giants, it is not advisable to stand back to back with one and call for a tape measure.

The authors' central thesis is that the broad principle needed to understand self-organization is already implicit in the second law of thermodynamics, and so has been right under our noses for a century and a half. Although the second law is a statement about increasing disorder, they argue that recent generalizations in non-equilibrium thermodynamics make it clear that it also plays a central role in creating order. The catchphrase they use to summarize this idea is "nature abhors a gradient". Being out of equilibrium automatically implies a gradient in the flow of energy from free energy to heat. For example, an organism takes in food, which provides the free energy needed to do work to perform its activities, maintain its form and reproduce. The conversion of free energy to entropy goes hand in hand with the maintenance of organization in living systems.

The twist is to claim that the need to reduce energy gradients drives a tendency towards increasing complexity in both living and nonliving systems. In their words: "Even before natural selection, the second law 'selects', from the kinetic, thermodynamic, and chemical options available, those systems best able to reduce gradients under given constraints." For example, they argue that the reason a climax forest replaces an earlier transition forest is that it is more efficient at fixing energy from the Sun, which also reduces the temperature gradient. They claim that the competition to reduce gradients introduces a force for selection, in which less effective mechanisms to reduce gradients are replaced by more effective ones. They argue that this is the fundamental reason why both living and non-living systems tend to display higher levels of organization over time.

This is an intriguing idea but I am not convinced that it makes sense. The selection process that the authors posit is never clearly defined, and they never explain why, or in what sense, it necessarily leads to increasing complexity. No one would dispute that the second law of thermodynamics is important for understanding the functioning of complex systems. Being out of equilibrium is a necessary condition for a physical phenomenon to display interesting complex behaviour, even if 'interesting' remains difficult to define. But the authors' claim that non-equilibrium thermodynamics explains just about everything falls flat. For 
example, consider a computer. No one would dispute that a power supply is essential. Even for a perfectly efficient computer, thermodynamics tells us that it takes at least $k T \ln 2$ energy units to erase a bit, where $T$ is the temperature and $k$ is the Boltzmann constant. But the need for power tells us nothing about what makes a laptop different from a washing machine. To understand how a computer works, and what it can and cannot do, requires the theory of computation, which is a logical theory that is disconnected from thermodynamics. The power supply can be designed by the same person who designs them for washing machines.

The key point is that, although the second law is necessary for the emergence of complex order, it is far from sufficient. Life is inherently an out-of-equilibrium phenomenon, but then so is an explosion. Something other than nonequilibrium thermodynamics is needed to explain why these are fundamentally different. Life relies on the ability of matter to store information and to implement functional relation- ships, which allow organisms to maintain their form and execute purposeful behaviours that enhance their survival. Such complex order depends on the rules by which matter interacts. It may well be that many of the details are not important, and that there are general principles that might allow us to determine when the result will be organization and when it will be chaos. But this cannot be understood in terms of thermodynamics alone.

Understanding the logical and physical principles that provide sufficient conditions for life is a fascinating and difficult problem that should keep scientists busy for at least a millennium. Thermodynamics clearly plays an essential part, and it is appropriate that the authors stress this - many accounts of the origin of life are easily rebutted on this point. But it isn't the principal actor, just one of many. The others remain unknown.

J. Doyne Farmer is at the Santa Fe Institute, 1399 Hyde Park Road, Santa Fe, New Mexico 87501, USA.

\section{Russia's secret weapons}

\author{
Biological Espionage: Special Operations of \\ the Soviet and Russian Foreign Intelligence \\ Services in the West \\ by Alexander Kouzminov \\ Greenhill: 2005. 192 pp. £12.99, \$19.95
}

\section{Jens H. Kuhn, Milton Leitenberg \& Raymond A. Zilinskas}

In 1992, President Boris Yeltsin admitted that the former Soviet Union had supported a secret biological-warfare programme, in violation of the Biological Toxin and Weapons Convention, which the Soviet Union ratified in 1975. Some of the researchers and officials who operated the programme, such as Ken Alibek, Igor Domaradskii and Serguei Popov, have provided personal accounts that shed light on the clandestine system. However, the compartmentalization and secrecy so prevalent in the former Soviet Union mean that such accounts describe only a fraction of the nation's bioweapons programme. Almost nothing is known about the biological-warfare activities of the Soviet ministries of defence, health and agriculture, the security agencies and the national academies.

As a result, any new information on the roles of these agencies in the Soviet bioweapons programme is welcomed by those who are concerned about whether Russia is continuing with its bioweapons programme. This is the backdrop to the publication of a book by Alexander Kouzminov, a former KGB agent, who claims to provide new and important information about the role of the KGB in the Soviet bioweapons programme. So, what do we learn from it?

Kouzminov describes himself as a former employee of the top-secret Department 12 of

\section{IMAGE UNAVAILABLE FOR COPYRIGHT REASONS}

In the dark: the bioweapons programme run from
KGB headquarters has remained largely secret.

Directorate S, the élite inner core of the KGB First Chief Directorate, which was responsible for operations abroad. One of the responsibilities of this department was to oversee 'illegals' - Russian intelligence operatives masquerading as Western nationals. Illegals were deployed to spy on Western biodefence activities, procure microbiological agents of interest for Soviet bioweapons research and development, and to perform acts of bioterrorism and sabotage. Kouzminov was a case handler for several illegals, including some that allegedly worked in a UK institute and at the World Health Organization (WHO). He repeatedly asserts that these illegals provided the Soviet Union with "significant" information.

Kouzminov does provide some information on his agency's work. He describes how Westerners were targeted for recruitment by the $\mathrm{KGB}$, and discusses the recruitment process and the means whereby data collected by agents and illegals were transported from the West to the Soviet Union. These procedures have previously been described by defectors and students of the Soviet intelligence system, and Kouzminov's book adds little to the story already in the public domain. Disappointingly, it provides almost no information on how the KGB transformed the data into intelligence, and how this was then used.

According to Kouzminov, individuals were deployed in the West and given numerous objectives related to spying on national programmes. For example, he describes a husbandand-wife team who, while operating a mock medical practice in Germany, were told by the KGB "to establish the locations of all NATO installations; their command personnel...air-force bases, and cruise-missile and rocket sites". It is doubtful that two individuals could accomplish all this. And Kouzminov's explanation that the KGB placed agents in the WHO to obtain information about the "development of vaccines against the most dangerous human and animal viral diseases" seems rather lame, given that anyone could obtain this information simply by telephoning WHO representatives.

The author further alleges that around 1980 a KGB agent was placed inside the US Army Medical Research Institute of Infectious Diseases at Fort Detrick, Maryland, and that another agent was employed by an unnamed British institute (probably the National Institute for Biological Standards and Control, which was not engaged in biodefence). What did these agents do? Did they provide information about US and UK defensive efforts that might be used by the Soviet bioweapons programme? Did they inform their superiors that neither country actually had an offensive programme? Perhaps they provided information on the development of vaccines that might have been useful to the Soviet defensive programme?

In fact, Kouzminov provides little information on the accomplishments of these and other agents in the biological field. Nor does he identify the Soviet research institutes with which the KGB allegedly collaborated in an effort to create more potent bioweapons, despite the fact that many of them are known today to Western security and academic communities.

Kouzminov describes himself as a biophysicist with a microbiological background, so it is surprising how many technical mistakes he makes. For example, he misidentifies the bacteria Bacillus anthracis and rickettsiae as viruses, and misspells agents such as Francisella tularensis and Yersinia pestis. 